\title{
Synthesis of Superconductor-Topological Insulator Hybrid Nanoribbon Structures
}

\author{
Piet Schönherr and Fengyu Zhang \\ Department of Physics, University of Oxford, Parks Road Oxford \\ OX1 3PU, UK \\ Vesna Srot and Peter van Aken \\ Stuttgart Center for Electron Microscopy, Max Planck Institute for \\ Solid State Research Heisenbergstr. 3, 70569 Stuttgart, Germany \\ Thorsten Hesjedal* \\ Department of Physics, University of Oxford \\ Parks Road Oxford, OX1 3PU, UK \\ thorsten.hesjedal@physics.ox.ac.uk \\ Received 31 March 2017 \\ Accepted 8 June 2017 \\ Published 3 August 2017
}

\begin{abstract}
Superconductors in proximity to topological insulators (TIs) have the potential to unlock exotic quantum phenomena, such as Majorana fermions. Quasi-one-dimensional structures are particularly suited to host these quantum states. Despite the growth of TI nanostructures being relatively straightforward, the in situ synthesis of superconductor-TI structures has been challenging. Here, we present a systematic study of the growth of the s-wave superconductor $\mathrm{Sn}$ on the $\mathrm{TI}_{\mathrm{Bi}} \mathrm{Te}_{3}$ by physical vapor transport. If $\mathrm{Sn}$ does not enter the $\mathrm{Bi}_{2} \mathrm{Te}_{3}$ lattice as a dopant, two types of structures are formed: $\mathrm{Sn}$ nanoparticles, that cover $\mathrm{Bi}_{2} \mathrm{Te}_{3}$ plates and belts in a cloud-like shape, and thin Sn layers on $\mathrm{Bi}_{2} \mathrm{Te}_{3}$ plates, that appear in puddle-like recessions. These heterostructures have potential applications as novel quantum devices.
\end{abstract}

Keywords: Topological insulators; chemical vapor deposition; heterostructures; superconductors; electron microscopy.

\section{Introduction}

Topological insulators (TIs) are band insulators with gapless, time-reversal symmetry protected topological surface states, which are not subject to backscattering by nonmagnetic impurities. ${ }^{1}$ Their bandstructure is characterized by an odd number of Dirac cones residing in the bulk bandgap. ${ }^{2}$ After the initial theoretical prediction, and the subsequent experimental demonstration of a two-dimensional

${ }^{*}$ Corresponding author.

This is an Open Access article published by World Scientific Publishing Company. It is distributed under the terms of the Creative Commons Attribution 4.0 (CC-BY) License. Further distribution of this work is permitted, provided the original work is properly cited. 
TI with chiral edge channels in the HgTe system, ${ }^{3}$ another class of TIs, the three-dimensional TIs $\mathrm{Bi}_{x} \mathrm{Sb}_{1-x},{ }^{4} \mathrm{Bi}_{2} \mathrm{Se}_{3}{ }^{5,6}$ and $\mathrm{Bi}_{2} \mathrm{Te}_{3}$ were experimentally confirmed. ${ }^{6,7}$ Breaking time-reversal symmetry in a TI introduces a bandgap at the Dirac point, opening up a range of exotic quantum phenomena, ${ }^{8}$ such as the topological magnetoelectric effect, ${ }^{9}$ magnetic monopoles, ${ }^{10}$ or the quantum anomalous Hall effect. ${ }^{11}$ In another related system, in topological superconductors, Majorana fermions were predicted. ${ }^{12-17}$ Majorana fermions are particles that are their own antiparticle. They emerge as bound states at flux vortices, e.g., in s-wave superconductors proximity-coupled to a TI through a two-dimensional state, resembling a spinless superconductor that does not break time-reversal symmetry. ${ }^{12}$ Anticipated applications of Majorana pairs include long lifetime quantum bits for quantum information processing. ${ }^{17}$ Topological Josephson-Witten effects are another class of emergent phenomena predicted to be observable in superconductor-TI-superconductor junctions. ${ }^{18}$

So far, a number of materials combinations for the observation of Majorana fermions involving onedimensional semiconductors, such as InAs nanowires, and superconductors have been theoretically proposed, ${ }^{19,20}$ and successfully experimentally studied. ${ }^{21-24}$ In contrast, the combination of TIs and superconductors in a heterostructure has been more challenging. A recent example is the combination of the two-dimensional TI HfTe $\mathrm{H}_{5}$ with the structurally compatible superconductor $\mathrm{HfTe}_{3} .{ }^{25}$ Alternatively, superconductors such as elemental $\mathrm{Sn}$ $\left(T_{\mathrm{C}} \approx 3.8 \mathrm{~K}\right)^{26}$ and $\mathrm{NbSe}_{2}\left(T_{\mathrm{C}} \approx 7.2 \mathrm{~K}\right)^{27,28}$ can be combined with the strong three-dimensional TIs $\mathrm{Bi}_{2} \mathrm{Te}_{3}$ or $\mathrm{Bi}_{2} \mathrm{Te}_{3}$, which have been studied in great detail. $^{7,29}$ The unit cell of these materials consists of three quintuple layers of composition $\mathrm{Se}(\mathrm{Te})$-Bi$\mathrm{Se}(\mathrm{Te})-\mathrm{Bi}-\mathrm{Se}(\mathrm{Te})$. The electronic properties of these semiconductors are largely dominated by defect-induced bulk carriers. The relative contribution of the topological surface state to the overall electronic transport properties can be greatly enhanced by high surface-to-volume ratios, inherent to nanobelts, ribbons, and plates. ${ }^{30,31}$ Further, nanoribbons proximity-coupled to s-wave superconductor on both surfaces have been theoretically shown to host robust topological insulating phases, independent of the position of the chemical potential. ${ }^{32}$ Nanostructures can be synthesized employing a number of techniques including solvothermal growth, ${ }^{30}$ molecular beam epitaxy, ${ }^{33}$ and physical vapor deposition. ${ }^{34-36,31}$

Here, we report the Sn-assisted physical vapor deposition growth of $\mathrm{Bi}_{2} \mathrm{Te}_{3}$ belts and ribbons on the nano- and sub-micron scale with the goal of producing superconductor-TI heterostructures. The morphology of the grown structures has been analyzed by scanning electron microscopy (SEM) and atomic force microscopy (AFM), and their chemical composition by energy dispersive X-ray (EDX) spectroscopy and Raman spectroscopy, in combination with powder X-ray diffraction (XRD). The structural details have been studied by high-resolution scanning transmission electron microscopy (STEM). Three types of $\mathrm{Sn}-\mathrm{Bi}_{2} \mathrm{Te}_{3}$ interactions, leading to different structures, can be distinguished: (i) Sn nanoparticle- $\mathrm{Bi}_{2} \mathrm{Te}_{3}$ structures, (ii) $\mathrm{Sn}$ puddles on $\mathrm{Bi}_{2} \mathrm{Te}_{3}$ plates, and (iii) Sn-doped $\mathrm{Bi}_{2} \mathrm{Te}_{3}$. In particular the $\mathrm{Sn}_{-} \mathrm{Bi}_{2} \mathrm{Te}_{3}$ heterostructures may be suitable hosts for Majorana fermions. The direct fabrication of heterostructures has the inherent advantage of fewer processing steps and well-defined interfaces, which makes them ideal testbeds for fundamental studies and future applications alike.

\section{Materials and Methods}

$\mathrm{Bi}_{2} \mathrm{Te}_{3}$ powder and $\mathrm{Sn}$ granules were placed in separate quartz boats at the center of a 1"-diameter quartz tube furnace (Nabertherm B180). Si(100) substrates (dimensions $1 \times 1 \mathrm{~cm}^{2}$ ) were solventcleaned, coated with the binding agent poly-l-lysine in DI water (1:10), washed, coated with a $\mathrm{TiO}_{2}$ nanoparticle solution, ${ }^{35}$ washed again, dried using a $\mathrm{N}_{2}$ blow gun, and placed downstream from the precursor. The furnace was subsequently heated up to $585^{\circ} \mathrm{C}$ at which the precursors vaporize. The vapor was transported to the substrates at the cold end $\left(\sim 480^{\circ} \mathrm{C}\right)$ by a laminar flow of $\mathrm{N}_{2}$. Sn-doped $\mathrm{Bi}_{2} \mathrm{Te}_{3}$ samples were achieved with a long growth time and relatively low flow rate $(8 \mathrm{~h}$ at $150 \mathrm{sccm})$. The density of structures tends to increase when a relatively large quantity of $\mathrm{Sn}$ is used, while also supplying the transport gas at a high flow rate (10 granules of $0.6 \mathrm{~g}$ each and $700 \mathrm{sccm})$. Samples in cross-sectional geometry were prepared by focused ion beam (FIB) for high-angle annular dark-field STEM (HAADF-STEM) and EDXSTEM at $200 \mathrm{kV}$ on a JEOL ARM200F (JEOL Co. Ltd), equipped with a cold field-emission gun and a DCOR probe Cs corrector (CEOS Co. 
Ltd.). EDX data were obtained using a $100 \mathrm{~mm}^{2}$ JEOL Centurio SDD-EDX detector (JEOL Co. Ltd.) and the Thermo Noran System 7 EDX system (Thermo Fisher Scientific Inc.). The TEM data was analyzed using Digital Micrograph (Gatan Inc.).

\section{Results and Discussions}

The $\mathrm{Sn}-\mathrm{Bi}_{2} \mathrm{Te}_{3}$ structures can be categorized into three types of systems, as discussed in the following sections.

Type (i) Sn nanoparticle- $\mathrm{Bi}_{2} \mathrm{Te}_{3}$ structures are characterized by Sn flakes that homogeneously cover all structures, as can be seen from a comparison of a Sn-free growth [c.f. Fig. 1(a)] and a Sn-assisted growth [c.f. Fig. 1(b)]. $\mathrm{TiO}_{2}$ catalyst clusters on the substrate can enhance the growth of Sn flakes.

$\mathrm{Bi}_{2} \mathrm{Te}_{3}$ plates and the tips of belts support the growth of larger $\mathrm{Sn}$ flakes that form cloud-like objects [Fig. 1(b), upper inset]. The density of flakes at the tip of the belt decreases to zero within a few microns away from the tip, and the base of the belt is free of Sn flakes. Plates, on the other hand, are fully covered by large Sn flakes, as can be seen in Fig. 1(b), lower inset. Sn is contained in clusters, and neither Bi nor Te react with the Sn flakes on top of $\mathrm{Bi}_{2} \mathrm{Te}_{3}$ plates [see Fig. 1(c)]. The SEM image shows white spots that cover the surface of a plate. Corresponding EDX elemental maps show homogeneous concentrations of $\mathrm{Bi}$ and $\mathrm{Te}$, but distinguished features of Sn that correspond to the flakes. The flakes are composed of $\sim 20$-nm-diameter $\mathrm{Sn}$ nanoparticles [cf. Fig. 1(d)]. If the coverage is lower the nanoparticles do not form flakes but stay rather separated as shown in Fig. 1(e).

Type (ii) Sn puddles on $\mathrm{Bi}_{2} \mathrm{Te}_{3}$ plates appear as white spots on a metallic surface under an optical microscope [see Fig. 2(a)]. The part near the base of the plate is nearly free of puddles, whereas the coverage at the top is very high, similar to the coverage of belts by Sn flakes. Most of the puddles have triangular shapes that are aligned with respect to each other [Fig. 2(b)]. This is most likely a
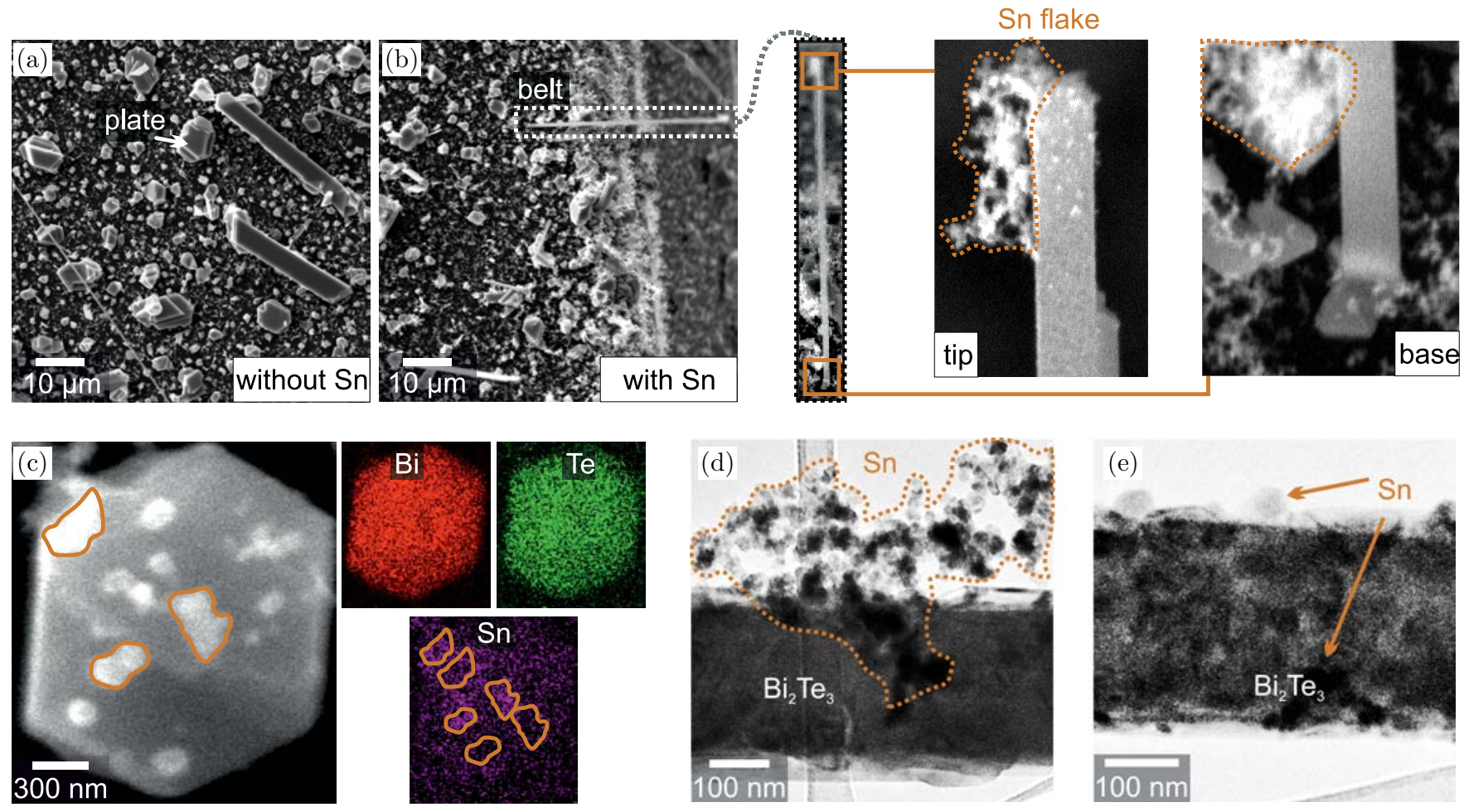

Fig. 1. Sn flakes on $\mathrm{Bi}_{2} \mathrm{Te}_{3}$ structures (type (i)). SEM comparison of the growth (a) without and (b) with Sn precursor. Flakes preferentially grow on $\mathrm{Bi}_{2} \mathrm{Te}_{3}$ structures such as plates and the tip of belts (upper inset). Their coverage decreases towards the base of the ribbons (lower inset). (c) SEM image of a hexagonal plate covered with flakes (left), and EDX maps showing the elemental composition for $\mathrm{Bi}, \mathrm{Te}$, and $\mathrm{Sn}$ (right). Bi and Te are homogeneously distributed, while $\mathrm{Sn}$ is concentrated in the flakes (areas of high concentration are indicated by yellow outlines). (d) TEM micrograph of a Sn flake (above) attached to a belt (below, darker). The flake consists of Sn nanoparticles. (e) TEM image of Sn nanoparticles (examples indicated by white arrows) attached to a $\mathrm{Bi}_{2} \mathrm{Te}_{3}$ belt (color online). 

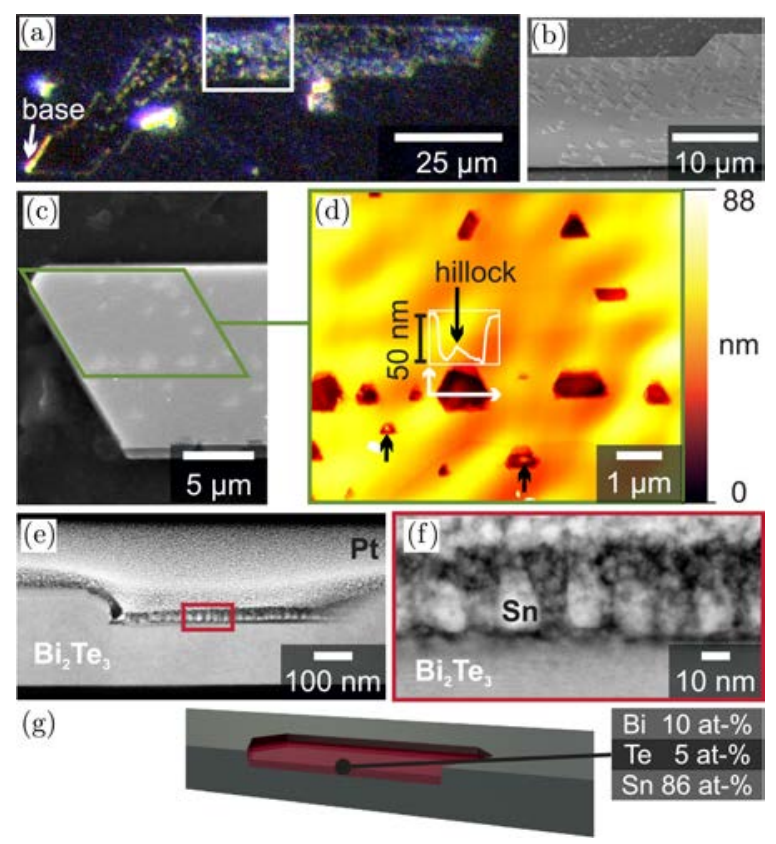

Fig. 2. Sn puddles on $\mathrm{Bi}_{2} \mathrm{Te}_{3}$ plates (type (ii)). (a) Optical microscope image of a pistol-shaped $\mathrm{Bi}_{2} \mathrm{Te}_{3}$ plate. $\mathrm{Sn}$ puddles appear as shiny spots. A white rectangle highlights the area in which the SEM micrograph in (c) was taken. (b) SEM image of triangular $\mathrm{Sn}$ puddles on $\mathrm{Bi}_{2} \mathrm{Te}_{3}$. (c) Tip of another $\mathrm{Bi}_{2} \mathrm{Te}_{3}$ ribbon. Puddles (cf. green outline) are aligned with the growth front (left) and the edge of the ribbon (top). (d) AFM image from the bottom right corner of the area marked in (c). The line profile (white) shows that a hillock is located on the bottom of the puddle. Other puddles show similar hillocks (see black arrows). (e) Cross-sectional STEM image of the plate shown in (b). The sample is covered by a protective Pt layer. (f) The high magnification image taken from the Sn-rich layer (indicated by a red rectangle) shows that the layer is composed of a periodic arrangement of Sn grains (bright). (g) Illustration of a Sn puddle (red) on $\mathrm{B} \mathrm{Bi}_{2} \mathrm{Te}_{3}$ plate (grey). The atomic composition of the $\mathrm{Sn}$ layer is indicated as measured by STEM EDX (color online). consequence of the underlying hexagonal crystal structure of $\mathrm{Bi}_{2} \mathrm{Te}_{3}$. Two lines of puddles in Fig. 2(c) form a parallelogram with the outline of the plate. This could be due to a growth mechanism that is related to the formation of Sn droplets at step edges of $\mathrm{Bi}_{2} \mathrm{Te}_{3}$. Indeed, we find a $20 \mathrm{~nm}$ hillock at the bottom of a puddle by AFM [cf. Fig. 2(d)]. This could be an initial droplet of Sn at a step edge around which further $\mathrm{Bi}_{2} \mathrm{Te}_{3}$ layers have grown. The puddle has a width of $1 \mu \mathrm{m}$ and a depth of $50 \mathrm{~nm}$.

An example of a puddle without a hillock is shown in Fig. 2(e) in cross-section prepared by FIB. Sn appears as a flat thin layer on top of the relatively thicker $\mathrm{Bi}_{2} \mathrm{Te}_{3}$ plate. The layer consists of $\sim 15 \times 20 \mathrm{~nm}$ sized $\mathrm{Sn}$ granules and is recessed beneath the top surface of the $\mathrm{Bi}_{2} \mathrm{Te}_{3}$ plate by $40 \mathrm{~nm}$. The edges of the layer above connect smoothly into the recession. Smaller granules fill up the space between the large Sn granules, as shown in Fig. 2(f) in a STEM micrograph. There is a residual concentration of $\mathrm{Bi}$ and $\mathrm{Te}$ found in this layer, as measured by STEM EDX and illustrated in Fig. 2(g).

Type (iii) Sn-doped $\mathrm{Bi}_{2} \mathrm{Te}_{3}$ structures typically have a Sn concentration of $\sim 5$ at. $\%$ as detected by EDX [Fig. 3(a)]. As compared to undoped $\mathrm{Bi}_{2} \mathrm{Te}_{3}$ (40 at.\% Bi and 60 at.\% Te), the doped structures have 36 at. $\% \mathrm{Bi}$ and 59 at.\% Te, which indicates that the $\mathrm{Sn}$ concentration comes at the expense of $\mathrm{Bi}$ if no additional phases such as SnTe are formed. We estimate the instrumental error for the SEM-EDX experiments to be $\pm 1 \%$ of the stated concentrations. In order to exclude the possibility of secondary phase formation, we transferred an ensemble of nanostructures from the as-grown substrate to a

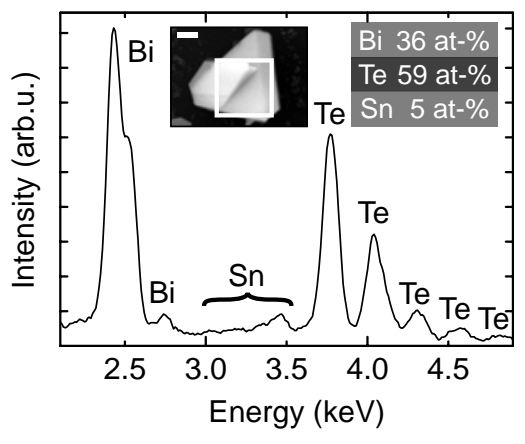

(a)

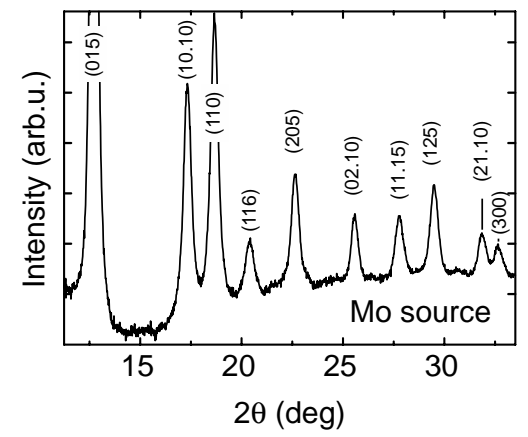

(b)

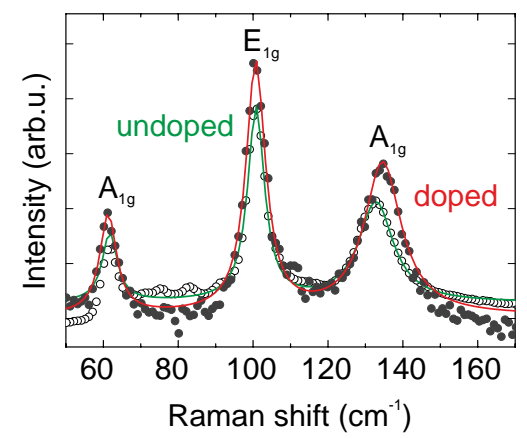

(c)

Fig. 3. Sn-doped $\mathrm{Bi}_{2} \mathrm{Te}_{3}$ nanostructures (type (iii)). (a) EDX spectrum of a plate. The inset shows an SEM image with the scan area marked by a white rectangle. The white scale bar corresponds to $1 \mu \mathrm{m}$. The Sn doping concentration is 5 at.\%. (b) Integrated powder XRD pattern taken by using a Mo X-ray source. The $\mathrm{Bi}_{2} \mathrm{Te}_{3}$ diffraction peaks are indexed. (c) Raman spectra of Sn-doped (dark filled dots, red fit) and undoped (empty dots, green fit) $\mathrm{Bi}_{2} \mathrm{Te}_{3}$. The data points are fitted using Lorentzian line shapes. The $\mathrm{A}_{1 \mathrm{~g}}$ mode is shifted by $2 \mathrm{~cm}^{-1}$ between the doped and the undoped sample (color online). 
micro-loop suitable for X-ray transmission experiments. The diffraction pattern resembles pure $\mathrm{Bi}_{2} \mathrm{Te}_{3}$ since $\mathrm{Bi}_{2} \mathrm{Te}_{3}$ peaks can be indexed based on standard diffraction data, ${ }^{37}$ as can be seen in Fig. 3(b). In particular, there are no extra peaks, i.e., additional phases. Sn causes a small lattice distortion as $(h k l)$-peaks with $h$ being nonzero are shifted by up to $0.2^{\circ}$ towards higher angles. Other peak positions can be matched with $\pm 0.1^{\circ}$ accuracy. These observations are consistent with mainly substitutional doping of Sn atoms on Bi lattice sites and a compression of the lattice. Interstitial doping at random places of the crystal lattice cannot be completely excluded because it would not affect the diffraction pattern.

In Fig. 3(c), we compare the Raman spectrum of pure $\mathrm{Bi}_{2} \mathrm{Te}_{3}$ (modes at $61.7 \mathrm{~cm}^{-1}, 100.8 \mathrm{~cm}^{-1}$, and $132.8 \mathrm{~cm}^{-1}$ ) with a Sn-doped sample. ${ }^{34}$ The $\mathrm{A}_{1 \mathrm{~g}}$ mode is slightly shifted. This is similar to Raman measurements on ternary $\left(\mathrm{Bi}_{1-x} \mathrm{Sb}_{x}\right)_{2} \mathrm{Te}_{3}$ compounds where the $A_{1 g}$ mode is most sensitive to changes in the concentration of Sb for small $x .^{38}$ It moves by $1.3 \%$ towards higher frequencies (from $132.8 \mathrm{~cm}^{-1}$ to $134.6 \mathrm{~cm}^{-1}$ ), since $\mathrm{Sn}$ is lighter than Bi. The two lower modes are shifted by less than $1 \%$ to lower frequencies which is within the instrumental error of the Raman system. Sn and Sb are next to each other in the periodic table with a relative mass difference of $2.5 \%$, so the energy of the lattice vibrations is nearly the same. Therefore, we used the literature data for $\left(\mathrm{Bi}_{1-x} \mathrm{Sb}_{x}\right)_{2} \mathrm{Te}_{3}$ and the procedure described in Ref. 34 to estimate the concentration of Sn causing the shift of the Raman line. ${ }^{38}$ We obtain a $\mathrm{Sn}$ concentration of $2 \%$ which is lower than the value from EDX measurements. In summary, $\mathrm{Sn}$ substitutes $\mathrm{Bi}$ in $\mathrm{Bi}_{2} \mathrm{Te}_{3}$ and reduces the $n$-type bulk carrier concentration of the material since it has one valence electron less than $\mathrm{Bi}^{39}$

Electronic transport measurements were carried out on type (i) $\mathrm{Sn}_{-} \mathrm{Bi}_{2} \mathrm{Te}_{3}$ hybrid structures. A long structure was selected and four silver paint contacts were applied, as shown in the inset to Fig. 4. The temperature-dependent resistance of the sample below $5 \mathrm{~K}$ is shown in Fig. 4. During cool-down, the resistance of the structure initially decreased as expected from the undecorated structures. ${ }^{31}$ At the superconducting critical temperature of Sn, however, a sudden increase in resistance of $\approx 1 \Omega$ is observed. Note that the density of Sn nanoparticles is below the percolation threshold, so the transport can be assumed to be through the ribbon. Further

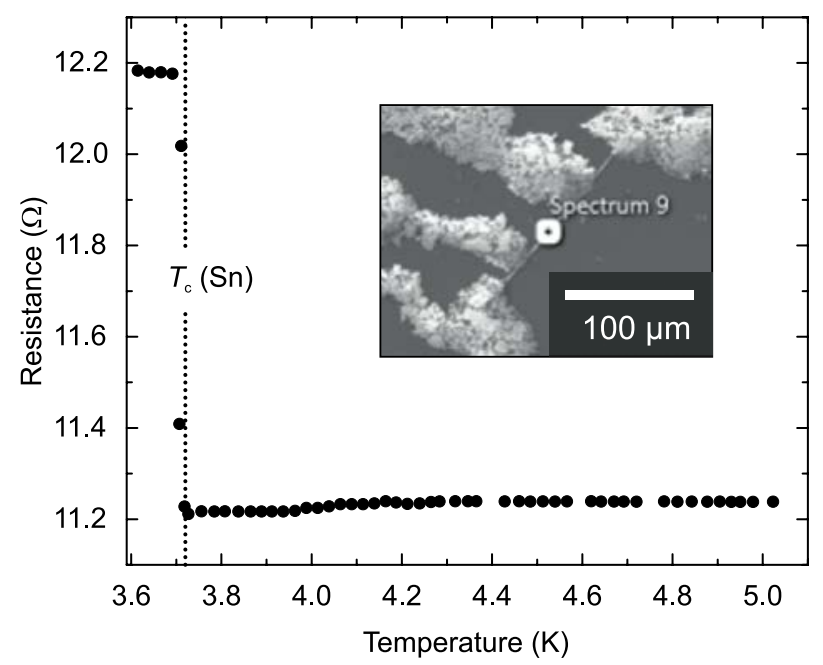

Fig. 4. Resistance of a type (i) structure as a function of temperature. The four-point contact measurement on a Sn$\mathrm{Bi}_{2} \mathrm{Te}_{3}$ hybrid structure (cf. inset) shows a jump in resistance at the superconducting transition temperature $\left(T_{\mathrm{C}}\right)$ of $\mathrm{Sn}$ of $\approx 3.72 \mathrm{~K}$ (color online).

studies are needed to understand this phenomenon in the framework of TI-superconductor proximity coupling.

\section{Conclusions}

We have performed a systematic growth study of the Sn-assisted synthesis of $\mathrm{Bi}_{2} \mathrm{Te}_{3}$ nano- and microstructures using physical vapor transport. Three different growth scenarios were observed depending on the growth conditions: cloud-like Sndecorated $\mathrm{Bi}_{2} \mathrm{Te}_{3}$, the local formation of Sn-rich areas on $\mathrm{Bi}_{2} \mathrm{Te}_{3}$ plates (puddles), and Sn-doped $\mathrm{Bi}_{2} \mathrm{Te}_{3}$. While the cloud-like $\mathrm{Sn}$ growth is related to tips and catalyst sites, the formation of Sn puddles is based on the spontaneous resublimation of $\mathrm{Sn}$ droplets on the surface of $\mathrm{Bi}_{2} \mathrm{Te}_{3}$. The different growth regimes are controlled by the $\mathrm{Sn}$ precursor quantity, carrier gas flux, and growth time. Each type has its specific application potentials. The high aspect ratio of nanoparticles - in combination with the controllable structure of a belt-like backbone as in the Sn-decorated structures - is desirable, e.g., for sensors. ${ }^{40-42}$ Superconductor-TI layered hybrid structures in the form of $\mathrm{Sn}$ puddles on $\mathrm{Bi}_{2} \mathrm{Te}_{3}$ are promising candidates for the observation and study of Majorana fermions, and ultimately topological quantum computation. ${ }^{25}$ First transport measurements show an interesting jump in resistance at the superconducting transition temperature of Sn. 
Finally, Sn-doped $\mathrm{Bi}_{2} \mathrm{Te}_{3}$ is suitable for making use of the topological surface state in spintronic devices due to the alignment of the chemical potential within the band gap of $\mathrm{Bi}_{2} \mathrm{Te}_{3}$. Therefore, $\mathrm{Sn}$ and $\mathrm{Bi}_{2} \mathrm{Te}_{3}$ for nanostructure growth is a very promising combination for future research and application.

\section{Acknowledgments}

We gratefully acknowledge Diamond Light Source for access to the Surfaces and Interfaces Laboratory. Funding from the European Union Seventh Framework Program under Grant Agreement 312483 - ESTEEM2 (Integrated Infrastructure Initiative I3) and transnational access to MPI-FKF (WP13) is acknowledged. P.S. acknowledges partial funding by EPSRC and Corpus Christi College (University of Oxford). This publication arises from research funded by the John Fell Oxford University Press (OUP) Research Fund. F.Z. acknowledges support by the University of Oxford's and USTC's summer internship program. $\mathrm{RCaH}$ is acknowledged for their hospitality.

\section{References}

1. X.-L. Qi and S.-C. Zhang, Phys. Today 63, 33 (2010).

2. H. Zhang, C.-X. Liu, X.-L. Qi, X. Dai, Z. Fang and S. C. Zhang, Nat. Phys. 5, 438 (2009).

3. M. Koenig, S. Wiedmann, C. Bruene, A. Roth, H. Buhmann, L. W. Molenkamp, X.-L. Qi and S.-C. Zhang, Science 318, 766 (2007).

4. D. Hsieh, D. Qian, L. Wray, Y. Xia, Y. S. Hor, R. J. Cava and M. Z. Hasan, Nature 452, 970 (2008).

5. D. Hsieh, Y. Xia, D. Qian, L. Wray, J. H. Dil, F. Meier, J. Osterwalder, L. Patthey, J. G. Checkelsky, N. P. Ong, A. V. Fedorov, H. Lin, A. Bansil, D. Grauer, Y. S. Hor, R. J. Cava and M. Z. Hasan, Nature 460, 1101 (2009).

6. Y. Xia, D. Qian, D. Hsieh, L. Wray, A. Pal, H. Lin, A. Bansil, D. Grauer, Y. S. Hor, R. J. Cava and M. Z. Hasan, Nat. Phys. 5, 398 (2009).

7. Y. L. Chen, J. G. Analytis, J.-H. Chu, Z. K. Liu, S.-K. Mo, X. L. Qi, H. J. Zhang, D. H. Lu, X. Dai, Z. Fang, S. C. Zhang, I. R. Fisher, Z. Hussain and Z.-X. Shen, Science 325, 178 (2009).

8. F. Wilczek, Phys. Scr. 168, 014003 (2016).

9. X.-L. Qi, T. L. Hughes and S.-C. Zhang, Phys. Rev. $B$ 78, 195424 (2008).

10. X.-L. Qi, R. Li, J. Zang and S.-C. Zhang, Science 323, 1184 (2009).

11. C.-Z. Chang, J. Zhang, X. Feng, J. Shen, Z. Zhang, M. Guo, K. Li, Y. Ou, P. Wei, L.-L. Wang, Z.-Q. Ji,
Y. Feng, S. Ji, X. Chen, J. Jia, X. Dai, Z. Fang, S.-C. Zhang, K. He, Y. Wang, L. Lu, X.-C. Ma and Q.-K. Xue, Science 340, 167 (2013).

12. L. Fu and C. L. Kane, Phys. Rev. Lett. 100, 096407 (2008).

13. F. Wilczek, Nat. Phys. 5, 614 (2009).

14. M. Z. Hasan and C. L. Kane, Rev. Mod. Phys. 82, 3045 (2010).

15. X.-L. Qi and S.-C. Zhang, Rev. Mod. Phys. 83, 1057 (2011).

16. P. W. Brouwer, Science 336, 989 (2012).

17. C. W. J. Beenakker, Annu. Rev. Condens. Matter Phys. 4, 113 (2013).

18. F. S. Nogueira, Z. Nussinov and J. van den Brink, Phys. Rev. Lett. 117, 167002 (2016).

19. R. M. Lutchyn, J. D. Sau and S. Das Sarma, Phys. Rev. Lett. 105, 077001 (2010).

20. Y. Oreg, G. Refael and F. von Oppen, Phys. Rev. Lett. 105, 177002 (2010).

21. V. Mourik, K. Zuo, S. M. Frolov, S. R. Plissard, E. P. A. M. Bakkers and L. P. Kouwenhoven, Science 336, 1003 (2012).

22. A. Das, Y. Ronen, Y. Most, Y. Oreg, M. Heiblum and H. Shtrikman, Nat. Phys. 8, 887 (2012).

23. M. T. Deng, C. L. Yu, G. Y. Huang, M. Larsson, P. Caroff and H. Q. Xu, Nano Lett. 12, 6414 (2012).

24. A. D. K. Finck, D. J. Van Harlingen, P. K. Mohseni, K. Jung and X. Li, Phys. Rev. Lett. 110, 126406 (2013).

25. Y.-Q. Wang, X. Wu, Y.-L. Wang, Y. Shao, T. Lei, J.-O. Wang, S.-Y. Zhu, H. Guo, L.-X. Zhao, G.-F. Chen, S. Nie, H.-M. Weng, K. Ibrahim, X. Dai, Z. Fang and H.-J. Gao, Adv. Mater. 28, 5013 (2016).

26. M. H. Beutel, N. G. Ebensperger, M. Thiemann, G. Untereiner, V. Fritz, M. Javaheri, J. Nägele, R. Rösslhuber, M. Dressel and M. Scheffler, Supercond. Sci. Technol. 29, 085011 (2016).

27. J.-P. Xu, M.-X. Wang, Z. L. Liu, J.-F. Ge, X. Yang, C. Liu, Z. A. Xu, D. Guan, C. L. Gao, D. Qian, Y. Liu, Q.-H. Wang, F.-C. Zhang, Q.-K. Xue and J.-F. Jia, Phys. Rev. Lett. 114, 017001 (2015).

28. H. H. Sun and J. F. Jia, Sci. China Phys. Mech. Astron. 60, 057401 (2017).

29. L. Fang, Y. Jia, D. J. Miller, M. L. Latimer, Z. L. Xiao, U. Welp, G. W. Crabtree and W.-K. Kwok, Nano Lett. 12, 6164 (2012).

30. F. Xiu, L. He, Y. Wang, L. Cheng, L.-T. Chang, M. Lang, G. Huang, X. Kou, Y. Zhou, X. Jiang, Z. Chen, J. Zou, A. Shailos and K. L. Wang, Nat. Nanotechnol. 6, 216 (2011).

31. P. Schönherr, F. Zhang, D. Kojda, R. Mitdank, M. Albrecht, F. S. Fischer and T. Hesjedal, Nanoscale Res. Lett. 11, 308 (2016).

32. P. Sitthison and T. D. Stanescu, Phys. Rev. B 90, 035313 (2014). 
33. S. E. Harrison, P. Schönherr, Y. Huo, J. S. Harris and T. Hesjedal, Appl. Phys. Lett. 105, 153114 (2014).

34. P. Schönherr, L. J. Collins-McIntyre, S. Zhang, P. Kusch, S. Reich, T. Giles, D. Daisenberger, D. Prabhakaran and T. Hesjedal, Nanoscale Res. Lett. 9, 127 (2014).

35. P. Schönherr, D. Prabhakaran, W. Jones, N. Dimitratos, M. Bowker and T. Hesjedal, Appl. Phys. Lett. 104, 253103 (2014).

36. Y. Guo, Z. Liu and H. Peng, Small 11, 3290 (2015).

37. A. Adam, Mater. Res. Bull. 42, 1986 (2007).
38. R. Richter and C. R. Becker, Phys. Stat. Sol. (b) 84, 619 (1977).

39. S. K. Kushwaha, Q. D. Gibson, J. Xiong, I. Pletikosic, A. P. Weber, A. V. Fedorov, N. P. Ong, T. Valla and R. J. Cava, J. Appl. Phys. 115, 143708 (2014).

40. Y. Zhang, J. Xu, P. Xu, Y. Zhu, X. Chen and W. Yu, Nanotechnology 21, 285501 (2010).

41. V. V. Dobrokhotov, D. N. McIlroy, M. Grant Norton, R. Abdelrahaman, A. Safir and C. A. Berven, Nanotechnology 20, 135504 (2009).

42. Y. Feng, I. S. Cho, P. M. Rao, L. Cai and X. Zheng, Nano Lett. 13, 855 (2013). 